\title{
Diabetes screening by telecentric digital holographic microscopy
}

\author{
Ana Doblas ${ }^{1 *}$, Enrique Roche ${ }^{2}$, F. Javier Ampudia-Blasco ${ }^{3}$, Manuel Martínez-Corral ${ }^{1}$, Genaro \\ Saavedra $^{1}$, and Jorge Garcia-Sucerquia ${ }^{4}$ \\ ${ }^{1}$ University of Valencia, 3D Imaging and Display Laboratory, Department of Optics, E-46100 Burjassot, Spain \\ ${ }^{2}$ University of Miguel Hernandez, Institute of Bioengineering, Department of Applied Biology-Nutrition, E-03203 \\ Elche, Spain \\ ${ }^{3}$ Valencia Clinic University Hospital, Department of Endocrinology and Nutrition, Diabetes Reference Unit, E-46010 \\ Valencia, Spain \\ ${ }^{4}$ Universidad Nacional de Colombia Sede Medellin, School of Physics, A.A: 3840-Medellin-050034-Colombia \\ *Corresponding author: telephone +34 963543936; fax + 34 963544715; e-mail address a.isabel.doblas@uv.es.
}

Key words: Digital holographic microscopy; phase retrieval; red blood cells; diabetes.

\section{Summary}

Diabetes is currently the world's fastest growing chronic disease and it is caused by deficient production of insulin by the endocrine pancreas or by abnormal insulin action in peripheral tissues. This results in persistent hyperglycemia that over time may produce chronic diabetic complications. Determination of glycated hemoglobin level is currently the gold standard method to evaluate and control sustained hyperglycemia in diabetic people. This measurement is currently made by high-performance liquid chromatography (HPLC), which is a complex chemical process that requires the extraction of blood from the antecubital vein. To reduce the complexity of that measurement, we propose a fully-optical technique that is based in the fact that there are changes in the optical properties of erythrocytes due to the presence of glucose-derived adducts in the hemoglobin molecule. To evaluate these changes, we propose to perform quantitative phase maps of erythrocytes by using telecentric digital holographic microscopy (DHM). Our experiments show that telecentric DHM allows detecting, almost in real time and from a single drop of blood, significant differences between erythrocytes of diabetic patients and healthy patients. Besides, our phase measurements are well correlated with the values of glycated hemoglobin and the blood glucose values.

\section{Introduction}

Diabetes mellitus $(\mathrm{DM})$ is a disease that includes a series of metabolic alterations that affect different organs and tissues (Maahs et al., 2010; Chen et al., 2011). In 2013, 382 million people around the world were affected with diabetes and according to the International Diabetes Federation (IDF) and the World Health Organization (WHO) this metabolic disorder is expected to affect 592 million people in 2035 (Maahs et al., 2010; Chen et al., 2011). Diabetes is mainly due to a deficient production of insulin by the 
endocrine pancreas or by abnormal insulin action in peripheral tissues. Diabetes is generally classified into four broad categories (Maraschin, 2012): type 1, type 2, gestational diabetes and others. Type 1 and type 2 are the most prevalent and therefore responsible for the majority of the health care cost attributed to this disease. Type 1 diabetes mellitus (T1DM) is characterized by the almost total loss of insulin due to an autoimmune destruction of pancreatic beta cells (Boitard, 2012). Therefore, type 1 diabetic patients require lifelong insulin injections, since there are no alternative hormones in the organism that can mimic the effect of insulin.

In any case, insulin injections cannot replace the exquisite control exerted by the pancreatic beta-cells that secrete the hormone in response to glucose variations in blood in a dose-dependent manner (Malik et al., 2014). For this reason, patients with T1DM present prominent fluctuations in blood glucose, with longer periods of hyperglycemia compared to non-diabetic individuals (Korytkowski, 2013; Lee, 2013; Todi, 2014). This may result over time in the development of secondary complications that affect mainly the retina, kidneys, nerves and cardiovascular system (Forbes et al., 2013). Therefore, hyperglycemia control is one of the main objectives to minimize the risk of developing retinopathy, nephropathy, neuropathy and cardiovascular complications (Korytkowski, 2013; Todi, 2014). Plasma glucose measurements based on dry chemistry and performed by a glucometer are quite accurate and minimally invasive, requiring only a drop of blood. However, these determinations, performed 4-6 times per day by the own patient, provide only a prompt index of the circulating glucose.

Long-term glycemic control is evaluated in terms of glycated hemoglobin (HbA1C) concentration (International Expert Committee, 2009). High glucose concentration in blood results in non-enzymatic glycation of side-chain amino groups of the amino acid residues, such as lysine, of circulating proteins. Glycation rate increases with higher concentrations of the sugar. The concentration of HbA1C indicates the level of exposure to persistent hyperglycemia over the last 2-3 months, coinciding with the half-life of hemoglobin (approximately 120 days). Therefore, HbA1C is the gold standard parameter to evaluate periodically glycemic control in patients with diabetes (International Expert Committee, 2009). HbA1C is measured currently by high-performance liquid chromatography (HPLC), and expressed both in \% or in International Federation of Clinical Chemistry (IFCC) units (mmol/mol) (Consensus Committed, 2007). Recently, the American Diabetes Association (ADA) as well as other institutional bodies have adopted HbA1C levels as diagnostic of diabetes if above $6.5 \%$ (American Diabetes Association, 2010). 
In this work, we propose a novel, fully-optical technique for the easy evaluation of HbA1C concentrations, and therefore for obtaining a fast screening of T1DM levels. This study is motivated by two previous published works (Mazarevica et al., 2002, Okamoto et al., 2000) which show that the refractive properties vary in presence of DM. In fact, Mazarevica et al. demonstrated a relationship between the refractive index, which is measured using a Nomarski polarizing-interference microscope, and $\mathrm{pH}$ level for diabetic and healthy donors. On the other hand, Okamoto et al. verified that variations in plasma glucose causes refractive index changes in eyes of diabetic subjects and, as a result, transient myopia or hyperopia is derived. Based on their findings, it seems logical to hypothesize that the phase of red blood cells (RBCs) could also change. Our method is based in the use of a telecentric DHM for the acquisition of quantitative phase maps of erythrocytes samples obtained from patients affected by diabetes, and also from non-diabetic (named as controls) individuals. After analyzing the alterations in the phase distributions we have found that there is a strong correlation between the absolute phase value of $\mathrm{RBCs}$ and the corresponding values of $\mathrm{HbA1C}$. This correlation permits us to suggest the use of telecentric DHM to quantify persistent hyperglycemia states. Besides, since only a small drop of blood is required and the phase measurement can be performed in any time, we can adopt this technique as a firstscreening method for the diagnosis of abnormal hyperglycemia .

\section{Material and methods}

\section{Sample preparation}

Samples were obtained from both non-diabetic individuals (controls) and also from patients with T1DM who have been under treatment with insulin injections over a period of $23 \pm 10$ years (mean \pm standard deviation). Capillary blood specimens from patients with T1DM were collected during routine ambulatory visits at the Diabetes Reference Center from the Clinic Hospital of Valencia (Spain). Control samples were obtained in a certified clinical analysis laboratory, Elche (Spain). All individuals participated voluntarily in this study, being adequately informed about the conditions and the purpose of the assessment. Participants signed an informed consent approved by the Ethics Committee of the University Miguel Hernández (Elche, Spain), fulfilling the requirements of the Declaration of Helsinki regarding human research.

Clinical measurements of HbA1C were performed using HPLC of blood extracted from the antecubital vein. On the other hand, telecentric DHM was performed, at the 3D Imaging and Display Laboratory 
(University of Valencia), on a capillary blood drop smearing on a glass slide. The smeared blood was dried out at room temperature in a dust-free environment. After the sample preparation, each slide was placed properly in the optical object path of our off-axis transmission telecentric DHM and a hologram was captured for each sample. The time required for the DHM operation and data processing did not exceed one minute per sample.

\section{Telecentric DHM}

The telecentric DHM was mounted, in open configuration, on an optical table. The scheme is shown in Figure 1. A He-Ne laser (wavelength $\lambda=633 \mathrm{~nm}, 50 \mathrm{~mW}$ output power, random polarization, by Edmund Optics) was used as the illumination source. The light proceeding from the laser was collimated and split to produce the reference $(R)$ and object waves $(O)$. A Nikon Plan Epi 50×/0.55 NA microscope objective (MO) and a tube lens (TL) of focal length $f_{T L}=200 \mathrm{~mm}$ were placed in the object arm of the microscope. A CCD camera with $1024 \times 1024$ square pixels of $6.9 \mu \mathrm{m}$ in side, recorded the holograms formed by the interference between the wavefield diffracted by the sample and a reference plane wave $R$. In order to operate in single-shot mode, the DHM was arranged in off-axis geometry by slightly tilting the reference wave $R$. The beam-splitter BS2 and the mirror M2 controlled the tilt angle. Note that the DHM operated in a telecentric regime in order to provide accurate quantitative phase measurements (Doblas et al., 2013; Doblas et al., 2014). A detailed description of the image formation procedure and the reconstruction process have been carefully given in Refs. (Doblas et al., 2014) and (Sánchez-Ortiga et al., 2014).

Since DHM is a holographic method, the computational reconstruction of the object wave follows wellestablished and exact methods. Specifically, to retrieve the object information, it is necessary to filter out the Fourier transform of the object information from the hologram spectrum, and then to perform the inverse Fourier transform. It is worth to mention that in our scheme the CCD is placed at the image plane of the imaging system, see Figure 1, and therefore not back-propagation algorithms are required (Kim, 2011; Kreis, 2004; Picart \& Li, 2012, Popescu, 2011). The computational processing of the digital holograms to provide a quantitative phase map of the sample was performed using Matlab (Mathworks, Natick, MA).

Stability analysis

As it is well known, phase measurements are strongly dependent on ambient fluctuations. To provide a controlled environment during the holograms acquisition, the complete microscope has been enclosed in a 
polymethymethacrylate (PMMA) cage with its temperature controlled. To exclude any incidence of ambient perturbations on the results of the experiment, we initially tested the stability of the set-up by recording twenty-five holograms of a test sample over a period of 5 minutes. From the twenty-five holograms, we calculated the corresponding twenty-five phase maps. Using these maps, the mean value of the phase over an area free of RBCs information was measured. It is worth to mention that the size of the area used to compute the mean phase was $41 \times 41 \mu \mathrm{m}^{2}$. For this region, the obtained value was 1.591 $\pm 0.013 \mathrm{rad}$. From the small value of the standard deviation $(0.013 \mathrm{rad})$, we concluded that our DHM system was fairly isolated from ambient fluctuations to guarantee trustable measurements for at least 5 minutes and, thus, any divergence between the measurements would be due to differences on the samples.

\section{Statistical analysis}

Non-parametric tests were used since the sample was small and, consequently, the variables were not normally distributed. Mann-Whitney (Hollander et al., 1999; Randolph et al., 2013) test was used to assess differences between control and diabetes groups with each one of the diagnostic methods (DHM and HPLC). Correlations between methods were later computed by means of the Spearman rho test $[23,24]$. Note that the statistical tests can be interpreted using p-values (Hollander et al., 1999; Randolph et al., 2013). The p-value is the probability of obtaining a test statistic result at least as extreme as the one that was actually observed, assuming that the null hypothesis is true. If the p-value is less than the required significance level, then one says the null hypothesis is rejected at the given level of significance. The lower significance level the stronger presumption against null hypothesis. All data were statistically analyzed using the SPSS software (version 22.0).

\section{Results and Discussion}

Table 1 summarizes the main characteristics of the population participating in the study. The total number of participants was 43 , separated into 14 controls ( 9 men and 5 women) and 29 T1DM patients (13 men and 16 women). The results shown in Table 1 indicate that blood glucose and HbA1C values of the control group are much more homogeneous, in contrast to the values obtained from the T1DM group.

Table 1 Basic characteristics (mean, standard deviation and range) of the sample under research.

Control $(\mathrm{N}=14) \quad$ T1DM Patient $(\mathrm{N}=29)$




\begin{tabular}{ccccccc}
\hline & Mean & $\begin{array}{c}\text { Standard } \\
\text { deviation }\end{array}$ & Range & Mean & $\begin{array}{c}\text { Standard } \\
\text { deviation }\end{array}$ & Range \\
& & & & & & \\
\hline Age (year) & 33 & 14 & $22-58$ & 41 & 8 & $21-55$ \\
Height (cm) & 170 & 10 & $151-190$ & 168 & 7 & $158-185$ \\
Weight (kg) & 74 & 15 & $53-97$ & 73 & 12 & $55.5-96.7$ \\
Glucose (mg/dl) & 85 & 12 & $63-120$ & 171 & 77 & $24-408$ \\
HbA1C (\%) & 5.2 & 0.3 & $4.8-6.0$ & 8.3 & 0.8 & $6.10-10.3$ \\
\hline
\end{tabular}

Using our telecentric DHM we measured the phase maps of RBC samples from both the healthy control group and the T1DM patients. As illustrating example of the results we obtained, in Figure 2 we show the phase maps corresponding to three controls and to three patients. For better visualization, the images have been pseudo-colored using the same color scale. From these samples, it is apparent that phase values of healthy RBCs are significantly smaller than those obtained for the T1DM group. The phase maps were used to calculate a mean phase value for each participant. For each RBC the phase was obtained as the average value over a square area of approximately $1.12 \times 1.12 \mu \mathrm{m}^{2}$ surrounding the centroid of the RBC. The phase values of 20 erythrocytes were averaged to obtain the phase value for each individual of the test. Owing to the sample preparation, the measured phase includes contributions not only from the RBCs but also from the plasma components. To remove the latter, the average value of the phase over the areas free of RBCs is subtracted from the total phase. This procedure was indeed applied to the phase maps show in Figure 2, hence the phase value over the RBCs is essentially provided by the optical path length along the blood cells. It is worth to mention that the stripe-like patterns in Figure 2 are due to the nonunlimited accuracy of the digital holographic microscope.

Figure 3 shows the histograms of the RBC phases from healthy individuals and for diabetic patients. The mean values were $3.15 \pm 0.13 \mathrm{rad}$ and $3.72 \pm 0.15 \mathrm{rad}$, respectively. Clearly, the phase values in the T1DM group were significantly higher than in the healthy control group. In addition, there is a big gap between the phase values of the two groups, see in Figure 3 that the phase values ranged $2.94-3.30 \mathrm{rad}$ for healthy RBCs and $3.51-4.01 \mathrm{rad}$ for T1DM RBCs. It is then reasonable to claim that the phase measurement made with telecentric DHM permits to divide the population into groups of healthy or T1DM patients and, moreover, phase values above $3.40 \mathrm{rad}$ are an indication of hyperglycemia. Another way to ensure this conclusion, and perhaps even the most formal way, is by applying the Mann-Whitney test, which tells us if the phase values from both groups are independent of each other. It is important to 
realize that this test shows greater efficiency than the regular t-test (Hollander et al., 1999; Randolph et al., 2013) on non-normal distributions, see in Figure 3 that our phase distributions are not Gaussian. Using the Mann-Whitney test, we found that the phase distributions of both groups are statistically independent with a very high significance level $(\mathrm{p}<0.001)$.

As a proof of the validity of the diabetes screening with telecentric DHM, we have contrasted the DHM results with the $\mathrm{HbA1C}$ values, which is the gold standard to assess diabetic patients. In Figure 4 we have plotted the phase vs. the $\mathrm{HbA1C}$ values for all the population under study. We measured the rho correlation coefficient to obtain $\mathrm{rho}=0.739$, with $\mathrm{p}<0.01$. The high correlation coefficient and the clear separation between the two populations confirm that both parameters may potentially be used to diagnose diabetes states as well as to evaluate long-term glycemic control in patients with diabetes. This result can be explained by accepting the findings by Barer et al. (Barer, 1952) which demonstrated that hemoglobin concentration is directly related to phase values. Although, from Figure 5, it seems that both DHM and HPLC should be individually sufficient to separate out the two populations, it is worth to highlight that using both approaches would allow for even better screening of DM. Besides, DHM technique could be used as the first method since only a capillary blood drop is needed, it can be performed in any time and the phase measurement is obtained in a fairly instantaneous way.

\section{Conclusions}

In this paper is demonstrated, for the first time to our knowledge, the use of telecentric DHM for diabetes screening. The utility of the DHM results from the possibility of providing quantitative phase maps as indicators of long-term blood sugar control similar to HbA1C. Although a strictly-medical research would require the analysis of much more individuals, our results should be viewed as a proof-of-concept study. In addition, the proposed method shows great potential as optional tool to screen for diabetes because: (1) it is a minimally invasive technique since only a small drop of capillary blood is needed; (2) it can be performed at any time and the results can be obtained almost in real time; and (3) it is a widefield technique which can be easily implemented in a conventional microscope (Sánchez-Ortiga et al., 2015) and can be used to analyze illnesses in which the refractive index or/and the morphology are distorted (Byun et al., 2012; Kemper et al., 2010; Park et al., 2008; Pavillon et al., 2012; Sridharan et al., 2015). 


\section{Acknowledgment}

Authors acknowledge J. Esteve for helping to find the control group, J. M. Adsuar Laboratory for providing the measurements of controls and T1DM patients with Type 1 diabetes followed at the Diabetes Reference Unit of Clinic Universiy Hospital of Valencia, Spain. This work was supported by the Ministerio de Economia y Competitividad, Spain (Grant DPI2012-32994) and the Generalitat Valenciana (Grant PROMETEOII/2014/072). E. Roche is member of the CIBEROBN (Fisiopatología de la Obesidad y la Nutrición CB12/03/30038) Instituto de Salud Carlos III, Spain. A. Doblas welcomes funding from University of Valencia through the predoctoral fellowship program "Atracció de Talent”. J. GarciaSucerquia acknowledges the support from Universidad Nacional de Colombia, Hermes grant 19384 and the Programa de Internacionalización del Conocimiento.

\section{References}

American Diabetes Association. (2010) Executive summary: standards of medical care in diabetes2010. Diabetes Care 33, S4-S10.

Barer, R. (1952) Interference microscopy and mass determination. Nature 169, 366-367.

Boitard, C. (2012) Pancreatic islet autoimmunity. Presse. Med. 41, e636-e650.

Byun, H. S. et al. (2012) Optical measurement of biomechanical properties of individual erythrocytes from a sickle cell patient. Acta Biomaterialia 8, 4130-4138.

Chen, L., Magliano, D. J. \& Zimmet, P. Z. (2011) The worldwide epidemiology of type 2 diabetes mellitus-present and future perspectives. Nat. Rev. Endocrinol. 8, 228-236.

Consensus Committed. (2007) Consensus statement on the worldwide standardization of the hemoglobin A1C measurement. Diabetes Care 30, 2399-2400.

Doblas, A., Sánchez-Ortiga, E., Martínez-Corral, M., Saavedra, G., Andrés, P. \& Garcia-Sucerquia, J. (2013) Shift-variant digital holographic microscopy: inaccuracies in quantitative phase imaging. Opt. Lett. 38, 1352-1354. 
Doblas, A., Sánchez-Ortiga, E., Martínez-Corral, M., Saavedra, G. \& Garcia-Sucerquia, J. (2014) Accurate single-shot quantitative phase imaging of biological specimens with telecentric digital holographic microscopy. J. Biomed. Opt. 19, 046022.

Forbes, J. M. \& Cooper, M. E. (2013) Mechanisms of diabetic complications. Physiol Rev 93, 137-188.

Hollander, M. \& Wolfe, D. A. (1999) Nonparametric Statistical Methods. Wiley, Chichester.

International Expert Committee. (2009) International Expert Committee report on the role of the A1C assay in the diagnosis of diabetes. Diabetes Care 32, 1327-1334.

Kemper, B. et al. (2010) Label-free quantitative cell division monitoring of endothelial cells by digital holographic microscopy. J. Biomed. Opt. 15, 036009 (2010).

Kim, M. K. (2011) Digital holographic microscopy: principles, techniques and applications. SpringerVerlag, Berlin.

Korytkowski, M. T. (2013) In-patient management of diabetes: controversies and guidelines. Indian J. Endocrinol. Metab. 17, S630-S635.

Kreis, T. (2004) Handbook of holographic interferometry: optical and digital methods. Wiley, Chichester.

Lee, Y. A. (2013) Diabetes care for emerging adults: transition from pediatric to adult diabetes care systems. Ann. Pediatr. Endocrinol. Metab. 18, 106-110.

Maahs, D. M., West, N. A., Lawrence, J. M. \& Mayer-Davis, E. J. (2010) Epidemiology of type 1 diabetes. Endocrinol. Metab. Clin. North Am. 39, 481-497.

Malik, F. S. \& Taplin, C. E. (2014) Insulin therapy in children and adolescents with type 1 diabetes. Paediatr. Drugs 16, 141-150 (2014).

Maraschin, J. de F. (2012). Classification of diabetes. Adv. Exp. Med. Biol. 771, 12-19 (2012).

Mazarevica, G., Freivalds, T. \& Jurka, A. (2002) Properties of erythrocyte light refraction in diabetic patient. J. Biomed. Opt. 7, 244-247. 
Okamoto, F., Sone, H., Nonoyama, T. \& Hommura, S. (2000) Refractive changes in diabetic patients during intensive glycaemic control. Br. J. Ophthalmol. 10, 1097-1102.

Park, Y. K., Diez-Silva, M., Popescu, G., Lykotrafitis, G., Choi, W., Feld, M. S. \& Suresh, S. (2008) Refractive index maps and membrane dynamics of human red blood cells parasitized by Plasmodium falciparum. PNAS 105, 13730-13735.

Pavillon, N., Kühn, J., Moratal, C., Jourdain, P., Depeursinge, C., Magistretti, P. J. \& Marquet, P. (2012) Early cell death detection with digital holographic microscopy. PLoS ONE 7, e30912.

Picart, P. \& Li, J.-C. (2012) Digital Holography. Wiley, Chichester.

Popescu, G. (2011) Quantitative Phase Imaging of Cells and Tissues. McGraw-Hill, New York.

Randolph, K. A. \& Myers, L. L. (2013) Basic statistics in multivariate analysis. Oxford University Press, Oxford.

Sánchez-Ortiga, E., Doblas, A., Saavedra, G., Martínez-Corral, M. \& Garcia-Sucerquia, J. (2014) Offaxis digital holographic microscopy: practical design parameters for operating at diffraction limit. Appl. Opt. 53, 2058-2066.

Sánchez-Ortiga, E., Doblas, A., Saavedra, G., Martínez-Corral, M. \& Garcia-Sucerquia, J. (2015) Microscope, method and computer program for obtaining quantitative study of transparent samples by digital holographic microscopy. Spanish patent ES 2534960 A1.

Sridharan, S., Macias, V., Tangella, K., Kajdacsy-Balla, A. \& Popescu, G. (2015) Prediction of prostate cancer recurrence using quantitative phase imaging," Sci. Rep. 5. doi: doi:10.1038/srep09976.

Todi, S. (2014) Glycemic control in critically ill: a moving target. Indian J. Crit. Care Med. 18, 229-233. 